\title{
Procalcitonina para el diagnóstico de sepsis neonatal de origen nosocomial
}

\author{
D. Pérez Solís ${ }^{\mathrm{a}}$, J.B. López Sastre ${ }^{\mathrm{a}}$, G.D. Coto Cotallo ${ }^{\mathrm{a}}$, M.A.A. Diéguez Junquera $^{\mathrm{b}}$, \\ E.M. Deschamps Mosquera ${ }^{\mathrm{b}}$ y M. Crespo Hernández ${ }^{\mathrm{a}}$ \\ aDepartamento de Pediatría. ' Servicio de Inmunología. Hospital Universitario Central de Asturias. Oviedo. España.
}

\section{Antecedentes}

La sepsis nosocomial supone una de las mayores preocupaciones en las unidades de neonatología y, dada la falta de especificidad de sus síntomas, se hacen necesarias pruebas complementarias muy fiables para orientar el diagnóstico. El objetivo de este estudio es evaluar la utilidad de la procalcitonina (PCT) para el diagnóstico de sepsis neonatal de origen nosocomial y comparar sus resultados con los marcadores de sepsis más utilizados.

\section{Pacientes y métodos}

Estudio prospectivo realizado en una unidad de cuidados intensivos neonatales. Se incluyeron 20 casos de sepsis nosocomial y 20 controles de entre 4 y 30 días de vida. Se midieron la PCT, proteína $\mathrm{C}$ reactiva (PCR), recuento leucocitario e indice de neutrófilos inmaduros/totales (indice $\mathbf{I} / \mathrm{T}$ ) en el momento de la sospecha de sepsis. Se calculó la sensibilidad, especificidad, valores predictivos y cocientes de probabilidades del positivo (CPP) y del negativo (CPN) de los marcadores de infección estudiados.

\section{Resultados}

PCT, PCR e índice I/T mostraron capacidad diagnóstica con áreas bajo la curva COR de $0,849,0,880$ y 0,884 , respectivamente, sin diferencias estadísticamente significativas. Los puntos de corte óptimos fueron: PCT $\geq 0,65 \mathrm{ng} / \mathrm{ml}$ (sensibilidad 85\%; especificidad 80\%; CPP 4,25; CPN 0,19), PCR $\geq 5 \mu \mathrm{g} / \mathrm{ml}$ (sensibilidad 80\%; especificidad 95\%; CPP 16; CPN 0,21) e índice $I / T \geq 0,03$ (sensibilidad $90 \%$; especificidad 75\%; CPP 3,6; CPN 0,13).

\section{Conclusiones}

La PCT puede ser una herramienta útil para el diagnóstico de sepsis nosocomial en neonatos, aunque es necesario disponer de estudios con mayor número de pacientes para poder comparar su rendimiento con el de otros marcadores de sepsis neonatal.

Palabras clave:

Sepsis/diagnóstico. Recién nacido. Marcadores biológicos. Calcitonina. Proteína C reactiva. Recuento de leucocitos.

\section{PROCALCITONIN FOR THE DIAGNOSIS OF NOSOCOMIAL NEONATAL SEPSIS}

\section{Background}

Nosocomial sepsis is a major problem in neonatal units. Because the clinical signs are nonspecific, highly reliable diagnostic markers are required to guide diagnosis. The aim of this study was to evaluate the utility of procalcitonin (PCT) as a diagnostic marker for nosocomial neonatal sepsis, and to compare the results of PCT with those of the most widely used laboratory tests for sepsis.

\section{Patients and Methods}

Twenty neonates with nosocomial sepsis and 20 controls aged 4-30 days were included in a prospective study performed in a neonatal intensive care unit. PCT, C-reactive protein (CRP), leukocyte count, and the immature-tototal neutrophil ratio ( $\mathrm{I} / \mathrm{T}$ ratio) were measured at onset of signs of infection. The sensitivity, specificity, and likelihood ratio for a positive (LR+) and a negative (LR-) result were calculated.

\section{Results}

PCT, CRP, and the I/T ratio discriminated septic from nonseptic patients. Their areas under the ROC curve were $0.849,0.880$, and 0.884 , respectively, with no statistically significant differences. Optimal cut-off values were: 
PCT $\geq 0.65 \mathrm{ng} / \mathrm{ml}$ (sensitivity $85 \%$, specificity $80 \%$, LR + 4.25, LR- 0.19), PCR $\geq 5 \mu \mathrm{g} / \mathrm{ml}$ (sensitivity $80 \%$, specificity $95 \%, \mathbf{L R}+16, \mathbf{L R}-0.21$ ), and $\mathrm{I} / \mathrm{T} \geq 0.03$ (sensitivity $90 \%$, specificity $75 \%$, LR +3.6, LR- 0.13 ).

\section{Conclusions}

PCT may be a useful marker for the diagnosis of nosocomial neonatal sepsis. Studies with larger samples are required to compare the accuracy of PCT with that of other markers of sepsis.

\section{Key words:}

Sepsis/diagnosis. Newborn. Biological markers. Calcitonin. C-reactive protein. Leukocyte count.

\section{INTRODUCCIÓN}

Las infecciones de transmisión nosocomial constituyen en la actualidad una de las mayores preocupaciones en las unidades de neonatología. Su frecuencia ha ido aumentando en las últimas décadas en relación con la mayor supervivencia de los recién nacidos de muy bajo peso. Estas infecciones aumentan la mortalidad y prolongan la estancia hospitalaria de los supervivientes, incrementando así los costes asociados a los cuidados neonatales $^{1}$. En España la incidencia de sepsis nosocomial se sitúa en 2,1 infectados por cada 100 ingresos en unidades neonatales, pero oscila entre el 1,3\% de los neonatos con peso al nacimiento igual o superior a $1.500 \mathrm{~g}$ y el 17,3\% en los de menos de $1.500 \mathrm{~g}^{2}$.

Los signos clínicos de sepsis neonatal nosocomial son muy inespecíficos, lo cual unido a su elevada incidencia obliga a tratar empíricamente a muchos neonatos con antibióticos intravenosos. Además, la presencia de flora hospitalaria multirresistente complica la elección del tipo de antibiótico. Por todo ello son precisas pruebas com-

\section{TABLA 1. Signos clínicos y factores de riesgo de infección considerados en el estudio}

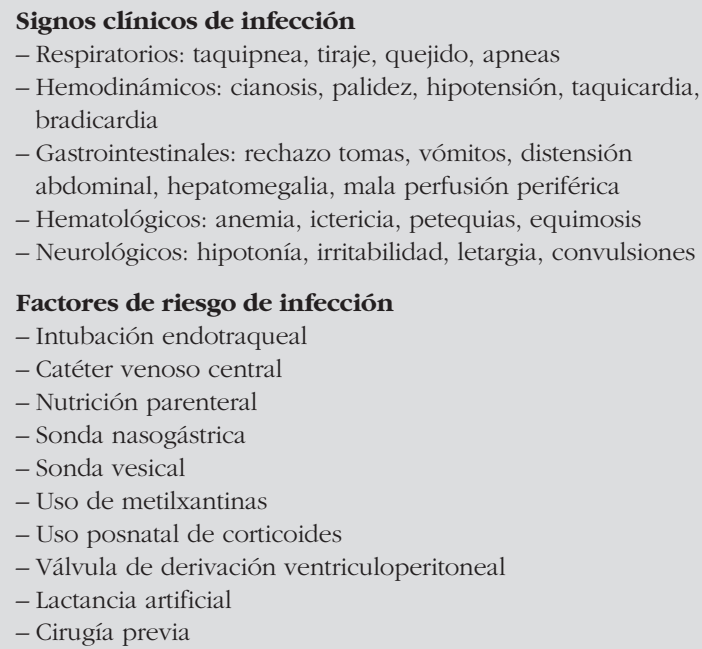

plementarias suficientemente sensibles y específicas para ayudar al clínico en la toma de decisiones.

En las últimas décadas se han estudiado diversos marcadores de infección neonatal, sobre todo índices leucocitarios y reactantes de fase aguda, algunos de los cuales se utilizan de manera habitual en la práctica clínica. Sin embargo, para la sepsis nosocomial existe comparativamente menos información sobre estos marcadores que para la sepsis de transmisión vertical, y ninguna prueba de laboratorio ha demostrado ser capaz de proporcionar un diagnóstico suficientemente fiable y precoz, por lo que se siguen buscando nuevos marcadores de infección ${ }^{3,4}$.

La procalcitonina (PCT) es la prohormona de la calcitonina. En condiciones normales sólo se sintetiza en pequeña cantidad en la glándula tiroides y en las células neuroendocrinas del pulmón, pero en la sepsis se produce en gran cantidad por casi todos los tejidos, aumentando de manera significativa sus concentraciones en sangre $^{5}$. Su inducción es rápida, detectándose a las 3 h tras un estímulo infeccioso, con pico a las $6 \mathrm{~h}$ y semivida de $24 \mathrm{~h}^{6}$. Por todo ello, la PCT ha sido evaluada como marcador de infección grave tanto en adultos como en niños, con resultados muy prometedores. Varios estudios recientes sugieren su utilidad para el diagnóstico precoz de sepsis neonatal precoz ${ }^{7-14}$ y tardía ${ }^{14-16}$.

El objetivo de este trabajo es estudiar la posible utilidad de la PCT para el diagnóstico de sepsis neonatal nosocomial, y comparar sus resultados con los de la proteína C reactiva (PCR), recuento leucocitario e índice de neutrófilos inmaduros/totales (índice I/T), que son los marcadores de infección más empleados en el momento actual.

\section{Pacientes y método}

Se realizó un estudio prospectivo en el Servicio de Neonatología del Hospital Universitario Central de Asturias entre febrero de 2000 y enero de 2002. Se incluyeron en el estudio los pacientes ingresados en la unidad de cuidados intensivos neonatales (UCIN) que reunían criterios de sepsis nosocomial y en quienes pudo realizarse la recogida adecuada de datos para el estudio. Por cada caso de sepsis se incluyó un control. Las extracciones del grupo de sepsis nosocomial fueron realizadas al establecerse la sospecha de infección. Para el grupo control se aprovecharon las extracciones programadas para estudios rutinarios. Se solicitó consentimiento informado escrito a los padres de los pacientes.

La sepsis nosocomial se definió de acuerdo con los criterios del Grupo de Hospitales Castrillo ${ }^{2,17}$. En recién nacidos con más de 4 días de vida se requerían al menos tres signos clínicos de infección (tabla 1) y hemocultivo positivo (excepto para gérmenes típicos de infección de transmisión vertical como Streptococcus agalactiae o Escherichia coli con idéntico resultado en cultivo de exudado vaginal materno, en cuyo caso se consideraba sepsis 
vertical tardía). En recién nacidos de hasta 4 dias de vida, dado que las sepsis son habitualmente de transmisión vertical, para considerarlas de tipo nosocomial se emplearon unos criterios muy estrictos que exigían al menos tres signos clínicos de infección (tabla 1), hemocultivo positivo a germen patógeno y evidencia de su origen nosocomial (aislamiento del mismo germen en material empleado en la atención del neonato o en personal sanitario, y ambiente epidémico de infección por dicho germen). En el caso de infecciones por estafilococo coagulasa-negativo se requerían dos hemocultivos periféricos positivos, o bien un solo hemocultivo cuando en las 2 botellas de cultivo se aislaba el mismo germen con idéntico patrón de antibiograma; en pacientes con catéter central se precisaba aislamiento del mismo germen con idéntico patrón de antibiograma en hemocultivo periférico y punta de catéter.

El grupo control estaba formado por recién nacidos de más de 4 días de vida con al menos un factor de riesgo de infección nosocomial (tabla 1) y ausencia de signos clínicos de infección.

La PCT se midió mediante análisis inmunoluminométrico (Lumitest ${ }^{\circledR}$ PCT, Brahms Diagnostica GmbH, Alemania), cuyo límite de detección se sitúa en 0,08 ng/ml. La PCR se determinó mediante turbidimetría (Turbitimer $^{\circledR}$, Dade Behring Marburg GmbH, Alemania), con un límite de detección de $5 \mu \mathrm{g} / \mathrm{ml}$. El recuento leucocitario se realizó mediante un sistema automatizado Coulter ${ }^{\circledR}$ STKS (Beckman Coulter, Inc., EE.UU.). Para la obtención del índice I/T se realizó recuento manual tras tinción Wright-Giemsa en el sistema Hema-Tek 2000 (Miles, Inc., EE.UU.).

Para el análisis estadístico se utilizó el programa informático SPSS 11.0 (SPSS Inc, 2001, EE.UU.) y Epidat 3.0 (Xunta de Galicia y Organización Panamericana de la Salud, 2003). En la parte analítica, dado que ni la PCT ni la PCR se ajustaron a la distribución normal, se emplearon pruebas no paramétricas. El nivel de significación estadística se estableció en $p<0,05$. Se calcularon la sensibilidad, especificidad, valores predictivos, índice J de Youden de seguridad diagnóstica (índice $\mathrm{J}=$ sensibilidad + especificidad -1 ) y cocientes de probabilidades con sus respectivos intervalos de confianza del 95\%, utilizando una hoja de cálculo proporcionada por el Programa de Habilidades en Lectura Crítica España (CASPe) disponible en: http://www.redcaspe.org/herramientas/.

\section{Resultados}

Fueron incluidos en el estudio 40 neonatos (20 casos de sepsis nosocomial y 20 controles) con una edad posnatal (media \pm desviación estándar) de 16,0 \pm 9,0 días (límites, 4-30 días). No se encontraron diferencias estadísticamente significativas entre casos y controles respecto al peso al nacimiento, edad gestacional, edad posnatal y distribución de sexos (tabla 2). Dentro de los

\section{TABLA 2. Comparación de distintas variables entre} los grupos del estudio

\begin{tabular}{|lccc|}
\hline & Controles & $\begin{array}{c}\text { Sepsis } \\
\text { nosocomial }\end{array}$ & $\mathbf{p}$ \\
\hline $\mathrm{n}$ & 20 & 20 & \\
Varones & $13(65,0 \%)$ & $14(70,0 \%)$ & $0,736^{*}$ \\
Edad gestacional & & & \\
$\quad$ (semanas) & $30,8 \pm 2,9$ & $30,8 \pm 3,7$ & $0,883^{* * *}$ \\
Peso (g) & $1.386 \pm 532$ & $1.544 \pm 718$ & $0,588^{* *}$ \\
Días de vida & $14,5 \pm 8,1$ & $17,6 \pm 9,8$ & $0,174^{* *}$ \\
\hline
\end{tabular}

Datos expresados como frecuencia (porcentaje) o como media \pm desviación estándar.

*Prueba de la chi cuadrado.

***Prueba U de Mann-Whitney.

TABLA 3. Comparación de las variables PCT, PCR, índice $\mathrm{I} / \mathrm{T}$ y recuento leucocitario entre los dos grupos

\begin{tabular}{|lcccc|}
\hline & PCT $(\mathbf{n g} / \mathbf{m l})$ & PCR $(\boldsymbol{\mu g} / \mathbf{m l})$ & Índice $\mathbf{I} / \mathbf{T}$ & Leucocitos/ $\boldsymbol{\mu l}$ \\
\hline Controles & 0,50 & $<5$ & 0,00 & 10.800 \\
& $(0,40-0,63)$ & $(<5-<5)$ & $(0,00-0,03)$ & $(8.200-16.375)$ \\
Sepsis & 13,46 & 25,60 & 0,08 & 11.350 \\
nosocomial & $(0,83-65,45)$ & $(9,45-58,37)$ & $(0,03-0,13)$ & $(7.825-16.100)$ \\
p* & 0,0002 & $<0,0001$ & $<0,0001$ & 0,892 \\
\hline
\end{tabular}

Los datos se expresan como mediana (rango intercuartil).

*Comparación mediante la prueba U de Mann-Whitney.

PCT: procalcitonina; PCR: proteína C reactiva; índice I/T: índice de neutrófilos inmaduros/totales.

20 pacientes con sepsis nosocomial se diagnosticaron 12 infecciones por Klebsiella pneumoniae, cuatro por Staphylococcus epidermidis, dos por Pseudomonas aeruginosa, una por Candida parapsilopsis y una infección mixta por Klebsiella pneumoniae y Enterococcus faecalis.

Los valores de PCT, PCR e índice I/T fueron significativamente superiores en el grupo de sepsis nosocomial respecto al grupo control (tabla 3), sin encontrarse diferencias estadísticamente significativas para el recuento leucocitario.

Se estudió la eficacia diagnóstica de PCT, PCR e índice I/T para el diagnóstico de sepsis nosocomial mediante sus curvas COR (fig. 1). Las áreas bajo la curva (intervalo de confianza del 95\%) fueron para la PCT 0,849 $(0,717-0,980)$, para la PCR $0,880(0,764-0,996)$ y para el índice I/T 0,884 $(0,780-0,988)$ sin encontrar diferencias estadísticamente significativas entre ellas. A partir de las curvas COR se obtuvieron los puntos de corte óptimos para calcular los índices de validez, que resultaron ser para la $\mathrm{PCT} \geq 0,65 \mathrm{ng} / \mathrm{ml}$, para la $\mathrm{PCR} \geq 5 \mu \mathrm{g} / \mathrm{ml}$ y para el índice $\mathrm{I} / \mathrm{T} \geq 0,03$. Las sensibilidades, especificidades, valores predictivos y cocientes de probabilidades para tales puntos de corte se recogen en la tabla 4. La PCR mostró un mayor rendimiento global entre sensibilidad y especificidad, aunque sin significación estadística. 


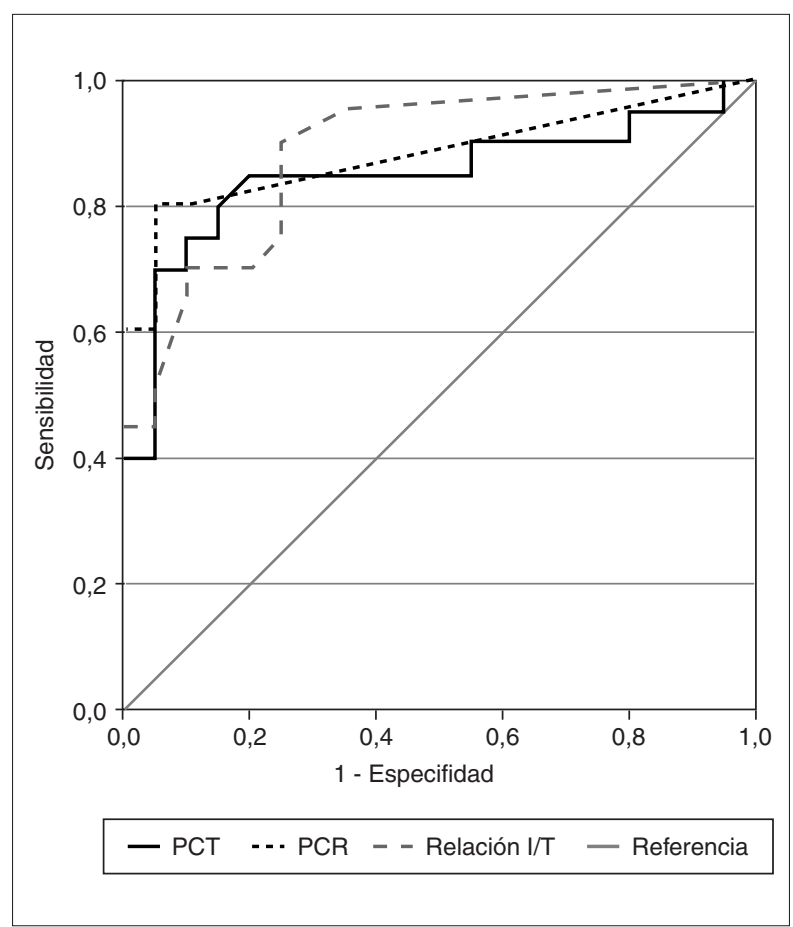

Figura 1. Curvas COR de PCT, PCR, e indice I/T para el diagnóstico de sepsis neonatal de origen nosocomial.

\begin{tabular}{|c|c|c|c|}
\hline Criterio & PCT $\geq 0,65 \mathrm{ng} / \mathrm{ml}$ & PCR $\geq 5,0 \mu \mathrm{g} / \mathrm{ml}$ & Indice $\mathrm{I} / \mathrm{T} \geq \mathbf{0 , 0 3}$ \\
\hline Sensibilidad & $\begin{array}{c}\mathbf{8 5 , 0} \% \\
(64,0-94,8)\end{array}$ & $\begin{array}{c}\mathbf{8 0 , 0} \% \\
(58,4-91,9)\end{array}$ & $\begin{array}{c}\mathbf{9 0 , 0} \% \\
(69,9-97,2)\end{array}$ \\
\hline Especificidad & $\begin{array}{c}\mathbf{8 0 , 0} \% \\
(58,4-91,9)\end{array}$ & $\begin{array}{c}\mathbf{9 5 , 0} \% \\
(76,4-99,1)\end{array}$ & $\begin{array}{c}\mathbf{7 5 , 0} \% \\
(53,1-88,8)\end{array}$ \\
\hline VPP & $\begin{array}{c}\mathbf{8 1 , 0} \% \\
(60-92,3)\end{array}$ & $\begin{array}{c}\mathbf{9 4 , 1 \%} \\
(73,0-99,0)\end{array}$ & $\begin{array}{c}\mathbf{7 8 , 3} \% \\
(58,1-90,3)\end{array}$ \\
\hline VPN & $\begin{array}{c}\mathbf{8 4 , 2} \% \\
(62,4-94,5)\end{array}$ & $\begin{array}{c}\mathbf{8 2 , 6 \%} \\
(62,9-93,0)\end{array}$ & $\begin{array}{c}\mathbf{8 8 , 2} \% \\
(65,7-96,7)\end{array}$ \\
\hline Índice J & 0,65 & 0,75 & 0,65 \\
\hline СРP & $\begin{array}{c}\mathbf{4 , 2 5} \\
(1,74-10,41)\end{array}$ & $\begin{array}{c}\mathbf{1 6 , 0 0} \\
(2,34-109,5)\end{array}$ & $\begin{array}{c}3, \mathbf{6 0} \\
(1,66-7,80)\end{array}$ \\
\hline $\mathrm{CPN}$ & $\begin{array}{c}\mathbf{0 , 1 9} \\
(0,06-0,54)\end{array}$ & $\begin{array}{c}\mathbf{0 , 2 1} \\
(0,09-0,51)\end{array}$ & $\begin{array}{c}\mathbf{0 , 1 3} \\
(0,04-0,51)\end{array}$ \\
\hline
\end{tabular}

Entre paréntesis se expresan los intervalos de confianza del 95\%. VPP: valor predictivo positivo; VPN: valor predictivo negativo; CPP: cociente de probabilidades del positivo; CPN: cociente de probabilidades del negativo; PCT: procalcitonina; PCR: proteína C reactiva; índice I/T: índice de neutrófilos inmaduros/totales.

\section{DisCuSión}

Así como la sepsis de transmisión vertical ha sido una preocupación constante en la neonatología, la importancia de la sepsis nosocomial ha ido aumentando a medida que los avances científicos han permitido una mayor supervivencia de recién nacidos grandes prematuros o aquejados de enfermedades graves. En la actualidad aún sigue existiendo un sesgo en la investigación sobre pruebas diagnósticas para las sepsis neonatales, de manera que son más numerosos los trabajos sobre sepsis de transmisión vertical o sepsis de inicio precoz que sobre sepsis nosocomiales ${ }^{3}$.

En nuestro estudio tanto la PCT como la PCR y el índice I/T mostraron capacidad diagnóstica para la sepsis neonatal de origen nosocomial, sin que pudiéramos demostrar lo mismo para el recuento leucocitario. Aunque el rendimiento de la PCR fue ligeramente superior, las diferencias encontradas no tuvieron significación estadística.

Existen pocas referencias en la literatura especializada con las cuales poder comparar nuestros resultados. Monneret et $a^{14}$ recogieron dentro de un estudio más amplio 14 casos de infección neonatal y 17 controles no infectados entre los 4 y 28 días de vida, pero -aunque los datos parecen indicar una notable eficacia diagnóstica y una elevación más rápida de la PCT respecto a la PCR- no proporcionan elementos suficientes para calcular sensibilidad y especificidad, etc. Poco después Chiesa et al ${ }^{8,16}$ publicaron los resultados de un estudio que incluía un subgrupo de infección nosocomial (3-30 días de vida) en el cual la PCT discriminó todos los casos de sepsis con sensibilidad y especificidad del 100\%; todos los casos de sepsis tenían concentraciones de PCT $\geq 2 \mathrm{ng} / \mathrm{ml}$ y todos los controles $\leq 1 \mathrm{ng} / \mathrm{ml}$. Más recientemente Enguix et al ${ }^{15}$ evaluaron la PCT para el diagnóstico de sepsis en recién nacidos de 3-30 días de vida ingresados en una UCIN, con sensibilidad del 98,6\% y especificidad del 88,9\% para concentraciones de PCT $\geq 8,05 \mathrm{ng} / \mathrm{ml}$, sin diferencias estadísticamente significativas respecto a la PCR y el amiloide sérico A.

Así como para las sepsis de transmisión vertical la utilización de la PCT está muy influenciada por la elevación fisiológica que se produce durante las primeras $48 \mathrm{~h}$ de vida $^{8,18}$, la habitual aparición más tardía de las sepsis nosocomiales permite obviar este fenómeno. El único condicionante significativo encontrado hasta ahora ha sido la distinta respuesta en los pacientes infectados por Staphylococcus epidermidis, con valores de PCT más bajos respecto al resto de agentes infecciosos ${ }^{16}$. En nuestro estudio el pequeño tamaño de muestra no permitió establecer comparaciones fiables entre distintos agentes infecciosos.

La principal limitación de este estudio reside en su escaso tamaño muestral, lo cual supone amplios intervalos de confianza en los indicadores del rendimiento diagnóstico. Esta limitación es común con todos los estudios sobre la PCT en sepsis neonatal nosocomial publicados hasta la fecha ${ }^{14-16}$, lo cual puede explicar las amplias variaciones en los puntos de corte seleccionados $(0,65-$ $8,05 \mathrm{ng} / \mathrm{ml}$ ). Tampoco es posible valorar las diferencias entre la eficacia de los distintos marcadores de infección, dado que para alcanzar la potencia estadística necesaria se 
precisan estudios con mucho mayor número de pacientes. Por otro lado, la selección de un control por cada caso de sepsis supone establecer una incidencia artificial de sepsis del $50 \%$ que no tiene por qué corresponderse con la real en los neonatos sometidos a chequeo de infección nosocomial. Por tanto, no se puede estimar la probabilidad preprueba y se introduce un sesgo en los valores predictivos. Otra limitación común con los estudios citados procede de la selección del grupo control, formado por pacientes asintomáticos. Este hecho supone una diferencia respecto a la utilización clínica de la prueba, cuya aplicación principal se daría en sujetos sintomáticos con el fin de apoyar o no el diagnóstico de infección. Por tanto se puede introducir un sesgo de selección que sobrevalore el rendimiento de la prueba, aunque tal sesgo afectaría a todos los marcadores estudiados, no sólo a la PCT.

Es importante señalar la heterogeneidad existente en los estudios sobre pruebas diagnósticas en cuanto a la definición de los casos de sepsis neonatal nosocomial. Una reciente conferencia internacional de consenso sobre sepsis pediátrica ha adaptado los criterios aceptados desde años en adultos a los distintos grupos de edad pediátricos, incluidos neonatos, lo cual facilitará la comparabilidad de futuras investigaciones ${ }^{19}$. No obstante, al haber sido realizado desde la perspectiva de las unidades de cuidados intensivos pediátricos, el consenso no incluye a los prematuros, principales protagonistas de las infecciones nosocomiales en las UCIN. Tampoco existe consenso sobre la diferenciación entre sepsis de transmisión vertical y sepsis nosocomial, que de manera habitual se limita a criterios cronológicos equiparándolas respectivamente a sepsis precoces y tardías. Esto supone ignorar la existencia de sepsis nosocomiales precoces y sepsis verticales tardías, que por su diferente perfil etiológico precisan de un enfoque terapéutico distinto.

Como conclusión, la PCT tiene capacidad discriminativa para la sepsis neonatal de origen nosocomial, por lo que podría ser útil como herramienta diagnóstica en la práctica clínica. Son necesarios estudios con mayor número de pacientes y una adecuada definición de los distintos grupos para establecer con precisión su verdadera utilidad y poder realizar comparaciones con otros marcadores como la PCR o los índices leucocitarios.

\section{Bibliografía}

1. Peter G, Cashore WJ. Infections acquired in the nursery: Epidemiology and control. En: Remington JS, Klein JO, editors. Infectious diseases of the fetus and newborn infant. Philadelphia: Saunders; 2001. p. 1264-83.

2. López Sastre JB, Coto Cotallo D, Fernández Colomer B. Neonatal sepsis of nosocomial origin: An epidemiological study from the Grupo de Hospitales Castrillo. J Perinat Med. 2002; 30:149-57.

3. Polin RA. The ins and outs of neonatal sepsis. J Pediatr. 2003; 143:3-4.

4. Weinberg GA, Powell KR. Laboratory aids for diagnosis of neonatal sepsis. En: Remington JS, Klein JO, editors. Infectious diseases of the fetus and newborn infant. Philadelphia: Saunders; 2001. p. 1327-44.

5. Müller B, White JC, Nylén ES, Snider RH, Becker KL, Habener JF. Ubiquitous expression of the calcitonin-i gene in multiple tissues in response to sepsis. J Clin Endocrinol Metab. 2001;86: 396-404.

6. Gendrel D, Bohuon C. Procalcitonin as a marker of bacterial infection. Pediatr Infect Dis J. 2000;19:679-87.

7. Guibourdenche J, Bedu A, Petzold L, Marchand M, MarianiKurdjian P, Marie F, et al. Biochemical markers of neonatal sepsis: Value of procalcitonin in the emergency setting. Ann Clin Biochem. 2002;39:130-5.

8. Chiesa C, Panero A, Rossi N, Stegagno M, De Giusti M, Osborn JF, et al. Reliability of procalcitonin concentrations for the diagnosis of sepsis in critically ill neonates. Clin Infect Dis. 1998;26: 664-72.

9. Gendrel D, Assicot M, Raymond J, Moulin F, Francoual C, Badoual J, et al. Procalcitonin as a marker for the early diagnosis of neonatal infection. J Pediatr. 1996;128:570-3.

10. Resch B, Gusenleitner W, Muller WD. Procalcitonin and interleukin-6 in the diagnosis of early-onset sepsis of the neonate. Acta Paediatr. 2003;92:243-5.

11. Lapillonne A, Basson E, Monneret G, Bienvenu J, Salle BL. Lack of specificity of procalcitonin for sepsis diagnosis in premature infants. Lancet. 1998;351:1211-2.

12. Chiesa C, Pellegrini G, Panero A, Osborn JF, Signore F, Assumma $\mathrm{M}$, et al. C-reactive protein, interleukin-6, and procalcitonin in the immediate postnatal period: Influence of illness severity, risk status, antenatal and perinatal complications, and infection. Clin Chem. 2003;49:60-8.

13. Maire F, Héraud MC, Loriette Y, Normand B, Bègue RJ, Labbé A. Intérêt de la procalcitonine dans les infections néonatales. Arch Pediatr. 1999;6:503-9.

14. Monneret G, Labaune JM, Isaac C, Bienvenu F, Putet G, Bienvenu J. Procalcitonin and C-reactive protein levels in neonatal infections. Acta Paediatr. 1997;86:209-12.

15. Enguix A, Rey C, Concha A, Medina A, Coto D, Diéguez MA Comparison of procalcitonin with C-reactive protein and serum amyloid for the early diagnosis of bacterial sepsis in critically ill neonates and children. Intensive Care Med. 2001;27: 211-5.

16. Chiesa C, Pacifico L, Rossi N, Panero A, Matrunola M, Mancuso G. Procalcitonin as a marker of nosocomial infections in the neonatal intensive care unit. Intensive Care Med. 2000;26:S175S7.

17. López Sastre J, Coto Cotallo GD, Ramos Aparicio A, Fernández Colomer B. Reflexiones en torno a la infección en el recién nacido. An Esp Pediatr. 2002;56:493-6.

18. Sachse C, Dressler F, Henkel E. Increased serum procalcitonin in newborn infants without infection. Clin Chem. 1998;44: 1343-4.

19. Goldstein B, Giroir B, Randolph A. International pediatric sepsis consensus conference: Definitions for sepsis and organ dysfunction in pediatrics. Pediatr Crit Care Med. 2005;6:2-8. 